\title{
ORTHOGONAL CLASSIFICATION OF HERMITIAN MATRICES
}

\author{
BY \\ LOO-KENG HUA
}

1. Introduction. Elsewhere $\left.{ }^{1}\right)$ the author established the symplectic classification of Hermitian matrices, which has applications to the geometry of symmetric matrices. It is the purpose of the present paper to treat the analogous problem: the orthogonal classification of Hermitian matrices. In other words, it may also be described as the quasi-unitary classification of symmetric matrices. Besides their own interest, the results of the present paper have applications in the geometries of skew-symmetric and symmetric matrices, as well as in the theory of automorphic functions of a matrix variable.

It should be noted that the method previously used is only applicable to the present problem for matrices of even order. In order to establish the general solution, we introduce here a different method.

Unless the contrary is stated, throughout the paper, capital latin letters denote $n$-rowed square matrices. Further let $M^{\prime}$ and $\bar{M}$ respectively denote the transposed matrix and conjugate imaginary matrix of $M$. I denotes the identity matrix.

2. Statement of the problems. Two Hermitian matrices $H$ and $K$ are said to be conjunctive orthogonally, if there is an orthogonal matrix $P$ such that

$$
P H P^{\prime}=K \text {. }
$$

From (1) and the orthogonality of $P$ we deduce immediately that

$$
P \bar{H} H P^{\prime}=\bar{K} K \text {. }
$$

Therefore, if two Hermitian matrices $H$ and $K$ are conjunctive orthogonally, the elementary divisors of the characteristic matrices of $\bar{H} H$ and $\bar{K} K$ are the same.

In the converse, two problems arise:

1. Does $\bar{H} H$ take any prescribed elementary divisors? The answer is in the negative. Then we ask further:

2. For a given admissible set of elementary divisors, is there a unique $H$, apart from orthogonal conjunctiveness, such that $\bar{H} H$ takes the given set as its elementary divisors?

The answer is also in the negative. More definitely, the elementary divisors of $\bar{H} H$ characterize the orthogonal conjunctiveness of $H$ with a reservation about the uncertainty of signs related to "signature."

Presented to the Society, February 23, 1946; received by the editors October 18, 1945.

(1) Amer. J. Math. vol. 66 (1944) pp. 531-563. 
More generally, apparently, we have the following problem. Two pairs $(H, S)$ and $\left(H_{1}, S_{1}\right)$ of matrices, where $H$ and $H_{1}$ are Hermitian and $S$ and $S_{1}$ are symmetric, are said to be equivalent if there exists a nonsingular matrix $P$ such that

$$
\bar{P} H P^{\prime}=H_{1},
$$

and

$$
P S P^{\prime}=S_{1}
$$

Consequently, we have

$$
\bar{P} H S^{-1} \overline{H S}^{-1} \bar{P}^{-1}=H_{1} S_{1}^{-1} \bar{H}_{1} \bar{S}_{1}^{-1},
$$

if $S$ is nonsingular. Hereafter we discuss only the case with nonsingular $S$. Analogously, we have also two problems.

3. Solution of problem 1.

THEOREM 1. Let $T$ be a symmetric matrix. The equation

$$
\bar{H} H=T
$$

is soluble in an Hermitian $H$, if and only if the elementary divisors of $T$ have the following two properties:

(i) Each elementary divisor corresponding to a negative characteristic root must occur an even number of times.

(ii) Complex elementary divisors must occur only in complex conjugate pairs.

Analogously, we have

THEOREM 1'. The equation

$$
B S^{-1} \overline{H S^{-1}}=Q
$$

is soluble in symmetric $S$ and Hermitian $H$ if and only if the elementary divisors of $Q$ satisfy (i) and (ii) of Theorem 1.

Equivalence of both theorems. If $S^{-1}=\Gamma \Gamma^{\prime}$ in (7), we have

$$
\left(\Gamma^{\prime} H \Gamma\right)\left(\Gamma^{\prime} \bar{H}\right)=\Gamma^{\prime} Q\left(\Gamma^{\prime}\right)^{-1} \text {. }
$$

Thus, Theorem $1^{\prime}$ implies Theorem 1 . Conversely, since we have $\Gamma$ such that $\Gamma Q\left(\Gamma^{\prime}\right)^{-1}=T$, Theorem 1 also implies Theorem $1^{\prime}$.

The sole difficulty lies in proving the property (i).

Proof of the theorems. (1) Suppose that

$$
T=\left(\begin{array}{ll}
q_{1} & 0 \\
0 & q_{2}
\end{array}\right)
$$

where $q_{1}$ and $\bar{q}_{2}$ have no characteristic root in common. Let 


$$
H=\left(\begin{array}{ll}
h_{1} & k \\
\bar{k}^{\prime} & h_{2}
\end{array}\right) .
$$

From (6), (9), and (10), we have

$$
\begin{aligned}
\bar{h}_{1} h_{1}+\bar{k} \bar{k}^{\prime} & =q_{1}, \\
h_{1} k+\bar{k} h_{2} & =0,
\end{aligned}
$$

and

$$
k^{\prime} k+\bar{h}_{2} h_{2}=q_{2} \text {. }
$$

From (11), (12) and (13), we deduce immediately

$$
\begin{aligned}
q_{1} \bar{k} & =\bar{h}_{1} h_{1} \bar{k}+\bar{k} \bar{k}^{\prime} \bar{k} \\
& =-\bar{h}_{1} k \bar{h}_{2}+\bar{k} \bar{k}^{\prime} \bar{k} \\
& =\bar{k}\left(h_{2} \bar{h}_{2}+\bar{k}^{\prime} \bar{k}\right)=\bar{k} \bar{q}_{2} .
\end{aligned}
$$

Since $q_{1}$ and $\bar{q}_{2}$ have no characteristic root in common, we have $k=0$. Thus (6) is soluble if and only if both equations

$$
h_{1} \bar{h}_{1}=q_{1}, \quad h_{2} \bar{h}_{2}=q_{2}
$$

are soluble in Hermitian $h_{1}$ and $h_{2}$. Thus we have to consider only either that $T$ has a real root or that $T$ has a pair of conjugate imaginary roots.

(2) Now we introduce the notations:

$$
j^{(p)}=\left(\begin{array}{ccccc}
0 & 0 & \cdots & 0 & 1 \\
0 & 0 & \cdots & 1 & 0 \\
\cdot & \cdot & \cdot & . & \cdot \\
1 & 0 & \cdots & 0 & 0
\end{array}\right)=\left(a_{i j}\right)
$$

where $a_{i j}=1$ if $i+j=p+1$ and 0 otherwise. Let $j_{\alpha}^{(p)}$ be the ordinary Jordan's canonical form $\left({ }^{2}\right)$ of a matrix with elementary divisor $(x-\alpha)^{p}$. Then $j j_{\alpha}$ is symmetric.

It is well known that there exists a real polynomial $f(x)$ such that

$$
f\left(j_{1}\right)^{2}=j_{1} .
$$

Let $s=\alpha^{1 / 2} j f\left(j_{1}\right)$ which is symmetric, since

$$
i j_{1}^{\infty}=j_{1}^{\prime} j_{1}^{\sigma-1}=\cdots=i_{1}^{\infty} j .
$$

It can be verified easily that $s$ satisfies

(2) For example, $j_{\alpha}^{(2)}=\left(\begin{array}{l}\alpha \\ 0 \\ 0\end{array}\right)$. 


$$
s j s=\alpha j j_{1} .
$$

(3) Now we consider the case that $T$ has only a pair of conjugate imaginary characteristic roots. Evidently the elementary divisors of $\bar{H} H-\lambda I$ and $H \vec{H}-\lambda I$ are the same; the solvability, therefore, implies the property (ii) of the theorem.

Conversely, the pair of symmetric matrices $(I, T)$ is congruent to a pair of matrices $\left(T_{1}, T_{2}\right)$ which is a direct sum of the following pairs:

$$
\left(\begin{array}{cc}
j & 0 \\
0 & i
\end{array}\right), \quad\left(\begin{array}{cc}
\alpha j j_{1} & 0 \\
0 & \bar{\alpha} j j_{1}
\end{array}\right) .
$$

Let

$$
\Gamma \Gamma^{\prime}=T_{1}, \quad \Gamma T \Gamma^{\prime}=T_{2}
$$

Then, (6) implies

$$
\left(\Gamma \bar{H} \Gamma^{\prime}\right) \bar{T}_{1}^{-1}\left(\Gamma H \Gamma^{\prime}\right)=T_{2}
$$

and conversely.

Since $T_{1}$ is real and $T_{1}{ }^{2}=I$, the solvability of (6) is equivalent to that of

$$
\bar{H} T_{1} H=T_{2} \text {. }
$$

Since the equation

$$
\left(\begin{array}{ll}
0 & s \\
s & 0
\end{array}\right)\left(\begin{array}{cc}
j & 0 \\
0 & j
\end{array}\right)\left(\begin{array}{ll}
0 & \bar{s} \\
s & 0
\end{array}\right)=\left(\begin{array}{cc}
\alpha j j_{1} & 0 \\
0 & \bar{\alpha} j j_{1}
\end{array}\right)
$$

is equivalent to $s j s=\alpha j j_{1}$, which is soluble in $s$ by (14), the theorem is proved for the case with complex characteristic roots.

(4) The easiest case is that in which $T$ has a positive root. Since we may assume that $\left(T_{1}, T_{2}\right)$ of $(3)$ is a direct sum of

$$
\left(j, \alpha j j_{1}\right), \quad \alpha>0,
$$

by (14), there exists a real $s$ such that

$$
s j s=\alpha j j_{1},
$$

and the theorem is now proved.

(5) Finally, there comes the difficult case that $\alpha$ is negative. Without loss of generality, we may assume that $\alpha=-1$.

Since, by (14), we have a symmetric matrix $s$ such that

$$
\left(\begin{array}{ll}
0 & s \\
s & 0
\end{array}\right)\left(\begin{array}{ll}
j & 0 \\
0 & j
\end{array}\right)\left(\begin{array}{ll}
0 & \bar{s} \\
s & 0
\end{array}\right)=\left(\begin{array}{cc}
-j j_{1} & 0 \\
0 & -j j_{1}
\end{array}\right),
$$

we see that if (i) is satisfied, (7) is soluble. The sole difficulty lies in the converse. 
(6) Now we write

$$
\begin{aligned}
& T_{0}=j^{\left(0_{1}\right)}+\cdots+j^{\left(\theta_{0}\right)}, \\
& T_{1}=j_{-1}^{\left(o_{1}\right)}+\cdots+j_{-1}^{\left(0_{0}\right)} .
\end{aligned}
$$

Let $K=H T_{0}$, then the equation

$$
\bar{H} T_{1} H=T_{0}
$$

takes the form

$$
\bar{K} T_{0} T_{1} K=I .
$$

From (19), we deduce immediately that

$$
K T_{0} T_{1}=K T_{0} T_{1} \bar{K} T_{0} T_{1} K=T_{0} T_{1} K .
$$

Let

$$
K=\left(k_{i j}\right), \quad k_{i j}=k_{i j}^{\left(0,0, q_{j}\right)} .
$$

From (20), we deduce

$$
k_{i j} j_{-1}^{\left(e_{j}\right)}=j_{-1}^{\left(e_{j}\right)} k_{i j}
$$

It is known that

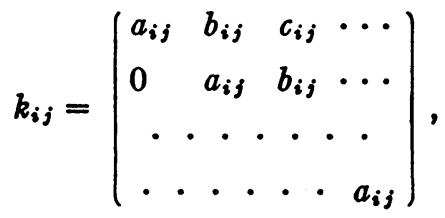

and in case $s_{j}>s_{k}$, we add $s_{j}-s_{k}$ rows of zeros below the matrix of the form (21) and in case $s_{j}<s_{k}$, we add $s_{k}-s_{j}$ columns of zeros before the matrix of the form (21).

From (19), we find

$$
\sum_{j} k_{i j j-1}^{\left(e_{j j}\right)} k_{j k}=\delta_{i k} I^{\left(\delta_{k}\right)}
$$

Now we consider the element at the $(1,1)$ position of the case $s_{i}=s_{k}$. By $(21)$,

$$
\sum_{j} k_{i j} k_{j k} j_{-1}^{\left(k_{j}\right)}=\delta_{i k} I^{\left(k_{k}\right)} \text {. }
$$

By the constitution of the first column of $j_{-1}$, we need only consider the element at the $(1,1)$ position of $k_{i j} k_{j k}$. It equals zero, if $s_{i}<s_{k}$ and if $s_{j}>s_{k}$. Thus the sum (23) runs only over all those $j$ 's with $s_{j}=s_{k}$.

Let $s$ be any integer occurring in the set $s_{1}, \cdots, s_{\text {. }}$. Without loss of generality, we may assume that

$$
s_{i}=s \text { for } 1 \leqq i \leqq m, \quad s_{i} \neq s \text { for } m<i \leqq e .
$$


Then, from (23), we deduce

$$
-\sum_{j=1}^{m} \bar{a}_{i j} a_{j k}=\delta_{i k}, \quad 1 \leqq i, k \leqq m,
$$

that is $\bar{A} A=-I^{(m)}$ if we let $A=\left(a_{i j}\right)_{1 \leq i, j \leq m}$. Taking determinants of both sides, we have $|d(A)|^{2}=(-1)^{m}$, which can not hold for odd $m$.

The theorem is now proved.

4. Square root of an orthogonal matrix. Now we require a result very likely due to Hilton, but his paper is not available here. Accordingly we state the rediscovered result in the following without proof.

We say a matrix $P$ is orthogonal with respect to a nonsingular symmetric matrix $S$, if

$$
P S P^{\prime}=S .
$$

In particular, for $S=I$, we omit the phrase "with respect to $I$."

Two matrices $A$ and $B$, orthogonal with respect to $S$, are said to be similar orthogonally, if there exists a matrix $P$ orthogonal with respect to $S$ such that $A=P B P^{-1}$.

THEOREM 2 (Hilton). Every orthogonal matrix is similar orthogonally to a direct sum of orthogonal matrices with elementary divisors either of the form

$$
(x-\alpha)^{r}, \quad(x-1 / \alpha)^{r},
$$

or of the form

$$
(x \pm 1)^{r},
$$

for odd $r$.

For the first case, the matrix orthogonal with respect to

$$
S=\left(\begin{array}{ll}
0 & I^{(r)} \\
I^{(r)} & 0
\end{array}\right)
$$

is similar to the matrix

$$
M=M^{(2 r)}=\left(\begin{array}{cc}
\left(j_{\alpha}^{(r)}\right)^{\prime} & 0 \\
0 & \left(j_{\alpha}^{(r)}\right)^{-1}
\end{array}\right)
$$

orthogonally, and for the second case, the matrix orthogonal with respect to

is similar to

$$
S_{1}=\left(\begin{array}{lll}
0 & 0 & I^{(p)} \\
0 & 1 & 0 \\
I^{(p)} & 0 & 0
\end{array}\right)
$$

$$
N=N^{(r)}=\left(\begin{array}{ccc}
\left(j_{ \pm 1}^{(p)}\right)^{\prime} & 0 & 0 \\
v & 1 & 0 \\
-\left(j_{ \pm 1}^{(p)}\right)^{-1} v^{\prime} v / 2 & -\left(j_{ \pm 1}^{(p)}\right)^{-1} v^{\prime} & \left(j_{ \pm 1}^{(p)}\right)^{-1}
\end{array}\right)
$$


orthogonally, where $v=(0,0, \cdots, 0,1)$ and $2 p+1=r$.

Evidently, the squares of the matrices $M$ and $N$ are matrices $M_{1}$ and $N_{1}$, orthogonal with respect to $S$ and $S_{1}$, with elementary divisors

$$
\left(x-\alpha^{2}\right)^{r}, \quad\left(x-\alpha^{-2}\right)^{r}
$$

and

$$
(x+1)^{r}
$$

respectively.

With a slight modification we have the following assertion: For given orthogonal $M_{1}$ and $N_{1}$ with elementary divisors (i') and (ii') respectively, we can find orthogonal matrices $M$ and $N$ with the elementary divisors (i) and (ii) respectively and with $\alpha$ in the right half-plane in the plane of complex numbers. Thus, except when $\alpha$ is negative, an orthogonal matrix has a square root matrix.

THEOREM 3. Let $R$ be an orthogonal matrix without a negative root and suppose that we have an Hermitian matrix $H$ such that

$$
R H=B \bar{R}^{\prime} \text {. }
$$

Then there exists an orthogonal matrix $Q$ with characteristic roots on the right half-plane such that $Q^{2}=R$ and

$$
Q H=H \bar{Q}^{\prime} \text {. }
$$

Proof. We may assume that $R$ is a direct sum of the matrices

$$
R=r_{1}+r_{2}+\cdots+r_{0}
$$

where $r_{i}$ has elementary divisors either of the form (i') or of the form (ii'). They are denoted typically by $M_{1}$ and $N_{1}$ respectively. We construct $M$ and $N$ accordingly; they are denoted by $q_{1}, \cdots, q_{e}$. Let

$$
Q=q_{1}+q_{2}+\cdots+q_{\bullet}
$$

Then, evidently, we have $Q^{2}=R$.

Further, from

$$
R H=H \bar{R}^{\prime},
$$

we have

$$
r_{i} h_{i j}=h_{i} \bar{r}_{j}^{\prime},
$$

and if this implies

$$
q_{i} h_{i j}=h_{i j} \bar{q}_{j}^{\prime},
$$

we have $Q H=H \bar{Q}^{\prime}$ and consequently we have the theorem.

In order to verify that (28) implies (29), we need only verify that 


$$
M^{2} X=X M^{2} \text { and } N^{2} X=X N^{2}
$$

implies

$$
M X=X M \text { and } N X=X N,
$$

respectively, where $M$ and $N$ are defined exactly as in (24) and (25). Straightforward calculation establishes both implications.

\section{Solution of the second problem.}

ThEOREM 4. If $H \bar{H}=K \bar{K}$, where $H$ and $K$ are both Hermitian, then there exist two matrices $P$ and $Q$ such that

and

$$
\bar{P} H P^{\prime}=\left(\begin{array}{ll}
h_{1} & 0 \\
0 & h_{2}
\end{array}\right)
$$

$$
\bar{Q} K Q^{\prime}=\left(\begin{array}{cc}
-h_{1} & 0 \\
0 & h_{2}
\end{array}\right)
$$
that

Proof. Since $K^{-1} H=R$ is orthogonal, we have an orthogonal matrix $T$ such

$$
T^{-1} R T=\left(\begin{array}{ll}
r_{1} & 0 \\
0 & r_{2}
\end{array}\right)=R_{1}
$$

where $r_{1}$ contains all negative characteristic roots of $R_{1}$. Then, we have

$$
H_{1}=K_{1} R_{1}=\bar{R}_{1}^{\prime} K_{1},
$$

where $H_{1}=\bar{T}^{\prime} H T, K_{1}=\bar{T}^{\prime} K T$ are both Hermitian. Since $r_{1}$ and $\bar{\gamma}_{2}$ have no characteristic root in common, we have

and

$$
K_{1}=\left(\begin{array}{cc}
k_{1} & 0 \\
0 & k_{2}
\end{array}\right)
$$

where

$$
H_{1}=\left(\begin{array}{ll}
h_{1} & 0 \\
0 & h_{2}
\end{array}\right)
$$

$$
k_{1} r_{1}=\bar{r}_{1}^{\prime} k_{1}
$$

and

$$
k_{2} r_{2}=\bar{r}_{2}^{\prime} k_{2} \text {. }
$$

By Theorem 3, we have an orthogonal $q_{2}$ such that $q_{2}{ }^{2}=r_{2}$ and

$$
h_{2}=k_{2} q_{2}^{2}=\bar{q}_{2}^{\prime} k_{2} q_{2} \text {. }
$$

Further we have an orthogonal matrix $q_{1}$ such that $q_{1}^{2}=-r_{1}$ and

$$
h_{1}=-k_{1} q_{1}^{2}=-\bar{q}_{1}^{\prime} k_{1} q_{1} \text {. }
$$

The theorem follows. 
6. Explicit result. The result previously obtained may be concluded in the following theorem.

TheOREM 5. Let $H$ be a nonsingular Hermitian matrix and $S$ be a nonsingular symmetrix matrix. The elementary divisors of the characteristic matrix of

$$
H S^{-1} \overline{H S^{-1}}
$$

are of the following three types:

$$
\begin{gathered}
(x-\alpha)^{\lambda}, \\
(x-\beta)^{\lambda}, \quad(x-\beta)^{\lambda},
\end{gathered}
$$

for real $\alpha>0$

and

$$
(x-\gamma)^{\lambda}, \quad(x-\bar{\gamma})^{\lambda},
$$

for complex $\boldsymbol{\gamma}$.

Let $\alpha_{1}=\alpha^{1 / 2}, \beta_{1}=i(-\beta)^{1 / 2}$ and $\gamma_{1}=\gamma^{1 / 2}$; the determination is taken on the right half-plane. Then the pair of matrices $(H, S)$ is equivalent to $\left(^{(}\right)$

$$
\left\{\begin{array}{l}
H_{1}=\sum_{\alpha}^{\prime}\left( \pm j j_{\alpha_{1}}\right)+\sum_{\beta}^{\prime}\left(\begin{array}{cc}
0 & j j_{\beta_{1}} \\
j j_{\beta_{1}} & 0
\end{array}\right)+\sum_{\gamma}^{\prime} \pm\left(\begin{array}{cc}
0 & j j_{\gamma_{1}} \\
j j_{\gamma_{1}} & 0
\end{array}\right) \\
S_{1}=\sum^{\prime}( \pm j)+\sum^{\prime}\left(\begin{array}{ll}
j & 0 \\
0 & j
\end{array}\right)+\sum^{\prime} \pm\left(\begin{array}{ll}
j & 0 \\
0 & j
\end{array}\right) .
\end{array}\right.
$$

In the theorem we have to justify only a point that the pair of matrices

$$
\left(\left(\begin{array}{cc}
0 & j j_{\beta_{1}} \\
j \bar{j}_{\beta_{1}} & 0
\end{array}\right),\left(\begin{array}{ll}
j & 0 \\
0 & j
\end{array}\right)\right)
$$

is equivalent to

$$
\left(-\left(\begin{array}{cc}
0 & j j_{\beta_{1}} \\
j j_{\beta_{1}} & 0
\end{array}\right), \quad-\left(\begin{array}{ll}
j & 0 \\
0 & j
\end{array}\right)\right) .
$$

In fact, we have a nonsingular matrix $\gamma$ such that $\gamma j j_{\beta_{1}} \gamma^{\prime}=-j j_{\beta_{1}}$ and $\gamma j \gamma^{\prime}=j$. Let

Then

$$
P=\left(\begin{array}{cc}
0 & i \bar{\gamma} \\
i \gamma & 0
\end{array}\right) .
$$

$$
\bar{P}\left(\begin{array}{cc}
0 & j j_{\beta_{1}} \\
j j_{\beta_{1}} & 0
\end{array}\right) P^{\prime}=-\left(\begin{array}{cc}
0 & j j_{\beta_{1}} \\
j j_{\beta_{1}} & 0
\end{array}\right)
$$

and

$$
P\left(\begin{array}{ll}
j & 0 \\
0 & j
\end{array}\right) P^{\prime}=-\left(\begin{array}{ll}
j & 0 \\
0 & j
\end{array}\right) .
$$

The situation of the indeterminate signs corresponding to a positive root (3) $\sum^{\prime}$ denotes direct sum. 
or to a pair of conjugate complex roots will be clarified by introducing the concept of signature system.

Suppose that $H S^{-1} \bar{H} \bar{S}^{-1}$ has an elementary divisor $(x-\alpha)^{\lambda}(\alpha>0)$ repeated $m$ times. Let $p$ and $q$ be the number of positive signs and negative signs appearing in the expression (31) corresponding to the elementary divisor $(x-\alpha)^{\lambda}$. Then $(p, q), p+q=m$, is called the signature corresponding to the elementary divisor $(x-\alpha)^{\lambda}$. Similarly we define the signature corresponding to

$$
(x-\gamma)^{\lambda}(x-\bar{\gamma})^{\lambda}
$$

for complex $\gamma$. The totality of elementary divisors and their corresponding signatures is called the elementary divisors with signature system of the pair of matrices $(H, S)$. Evidently, $(H, S)$ and $\left(H_{1}, S_{1}\right)$ are equivalent, if they have the same elementary divisors with the same signature system. The converse of this statement is also true. The proof of this fact can be constructed by adapting the results to be obtained in $\$ \$ 8-10$ and the method used in (6) of $\$ 3$. Owing to the similarity, we give here no details of the proof.

7. Anti-involutions. An orthogonal matrix $T$ satisfying

$$
T \bar{T}=I
$$

is called an anti-involution of the first kind, and that satisfying

$$
T \bar{T}=-I
$$

is called an anti-involution of the second kind.

Since $T T^{\prime}=I$, it is evident that the involution $T$ of the first kind is an Hermitian matrix. By Theorem 5, we have an orthogonal matrix $P$ such that

$$
P T \bar{P}^{\prime}=[1, \cdots, 1,-1, \cdots,-1]
$$

which is a diagonal matrix.

In case of the second kind, $i T$ is Hermitian, the roots of

$$
|(i T)(\overline{i T})-\lambda I|=0
$$

are all equal to -1 . The case can only happen for $n$ even. There exists an orthogonal matrix $P$ such that

where $n=2 p$.

$$
P(i T) \bar{P}^{\prime}=\left(\begin{array}{cc}
0 & i I^{(p)} \\
-i I^{(p)} & 0
\end{array}\right),
$$

8. Automorphs. Now we are going to study the group formed by all the matrices $P$ satisfying

$$
P S P^{\prime}=S
$$

and

$$
\bar{P} H P^{\prime}=H \text {. }
$$


By Theorem 5, we may write

$$
\begin{aligned}
& S=S_{1}^{(p)}+S_{2}^{(q)}, \\
& H=H_{1}^{(p)}+H_{2}^{(q)},
\end{aligned}
$$$$
p+q=n,
$$

where the two matrices

$$
H_{1} S_{1}^{-1} \bar{H}_{1} \bar{S}_{1}^{-1} \text { and } H_{2} S_{2}^{-1} \bar{H}_{2} \bar{S}_{2}^{-1}
$$

have no characteristic root in common. From (34) and (35), we have

$$
\bar{P} H S^{-1}=H S^{-1} P \text {. }
$$

It follows immediately that $P$ is decomposable as

$$
P=\left(\begin{array}{cc}
P_{1}^{(p)} & 0 \\
0 & P_{2}^{(q)}
\end{array}\right)
$$

Therefore, without loss of generality, we treat the problem with (i) $H S^{-1} \bar{H} \bar{S}^{-1}$ having only a pair of conjugate roots, (ii) $H S^{-1} \bar{H} \bar{S}^{-1}$ having only a positive root, and (iii) $H S^{-1} \bar{H} \bar{S}^{-1}$ having only a negative root.

We shall discuss the cases separately.

9. The case with a pair of complex roots. Now, without loss of generality, we take

$$
S=\sum_{1 \leq i \leq 0^{\prime}} \pm\left(\begin{array}{cc}
j^{\left(s_{i}\right)} & 0 \\
0 & j^{\left(s_{i}\right)}
\end{array}\right)
$$

and

$$
H=\sum_{1 \leqq i \leqq}^{\prime} \pm\left(\begin{array}{cc}
0 & j j_{\gamma}^{\left(s_{i}\right)} \\
j \bar{j}_{\gamma}^{\left(e_{i}\right)} & 0
\end{array}\right)
$$

where $s_{1} \geqq s_{2} \geqq \cdots \geqq s_{\text {e. }}$ Putting

$$
J^{(p)}=\sum_{1 \leq i \leq e}^{\prime}\left( \pm j^{\left(e_{i}\right)}\right)
$$

and

$$
J_{\gamma}^{(p)}=\sum_{1 \leq i \leq e}^{\prime}\left( \pm j j_{\gamma}^{\left(s_{i}\right)}\right),
$$

we treat, without loss of generality, the case that

$$
S=\left(\begin{array}{ll}
J & 0 \\
0 & J
\end{array}\right), \quad H=\left(\begin{array}{ll}
0 & J_{\gamma} \\
\bar{J}_{\gamma} & 0
\end{array}\right) .
$$

Let

$$
P=\left(\begin{array}{ll}
A & B \\
C & D
\end{array}\right), \quad A=A^{(p)},
$$


and so on. Then, from (36), we have

$$
\left(\begin{array}{cc}
\bar{A} & \bar{B} \\
\bar{C} & \bar{D}
\end{array}\right)\left(\begin{array}{cc}
0 & J_{\gamma} J \\
\overline{J_{\gamma} J} & 0
\end{array}\right)=\left(\begin{array}{cc}
0 & J_{\gamma} \\
\overline{J_{\gamma}} \bar{J} & 0
\end{array}\right)\left(\begin{array}{ll}
A & B \\
C & D
\end{array}\right)
$$

that is

$$
\begin{array}{ll}
\overline{B J}_{\gamma}=J_{\gamma} J C, & \bar{A} J_{\gamma} J=J_{\gamma} J D, \\
\overline{D J}_{\gamma} J \bar{J}_{\gamma} J A, & \bar{C} J_{\gamma} J=\bar{J}_{\gamma} J B .
\end{array}
$$

Then, we have

$$
B\left(J_{\gamma} J\right)^{2}=\left(\bar{J}_{\gamma} J\right) \bar{C}\left(J_{\gamma} J\right)=\left(\bar{J}_{\gamma} J\right)^{2} B .
$$

It follows that $B=0$ since the characteristic root of $\left(J_{\gamma} J\right)^{2}$ is equal to $\gamma^{2}$ and $\gamma^{2} \neq \bar{\gamma}^{2}$. Consequently $C=0$.

Further, from (37), we have

$$
A\left(\bar{J}_{\gamma} J\right)^{2}=\left(\bar{J}_{\gamma} J\right)^{2} A,
$$

and

$$
D=\left(J_{\gamma} J\right)^{-1} \bar{A}\left(J_{\gamma} J\right)
$$

From (34) we deduce

$$
A J A^{\prime}=J \text {. }
$$

Conversely, for any $A$ satisfying (38) and (40), the matrix

$$
P=\left(\begin{array}{cc}
A & 0 \\
0 & \left(J_{\gamma} J\right)^{-1} \bar{A}\left(J_{\gamma} J\right)
\end{array}\right)
$$

satisfies our requirement.

Then we need to find those $A$ satisfying both (38) and (40).

Let

$$
A=(I+Q)(I-Q)^{-1}
$$

(the exceptional case $|I+A|=0$ is negligible in counting of parameters).

From (38) and (40), we have

$$
Q\left(\bar{J}_{\nu} J\right)^{2}=\left(\bar{J}_{\gamma} J\right)^{2} Q
$$

and

$$
Q J+J Q^{\prime}=0 .
$$

Let $Q J=K$, which is a skew symmetric matrix and which satisfies

$$
K\left(J \bar{J}_{\gamma}\right)^{2}=\left(J \bar{J}_{\gamma}\right)^{2} K .
$$

(Notice that $J J_{\gamma} J=J_{\gamma^{\prime} \text {.) }}^{\prime}$

Putting

$$
K=\left(k_{i j}\right)_{1 \leq i, j \leq o,} \quad k_{i j}=k_{i j}^{\left(\theta_{i j}, \theta_{j}\right)},
$$


and

$$
k_{i j}=-k_{j i}^{\prime}
$$

we have

$$
k_{i j}\left(j_{\gamma}^{\left(e_{j}\right)}\right)^{2}=\left(j_{\gamma}^{\left(e_{j}\right)}\right)^{\prime 2} k_{i j}
$$

It follows immediately that

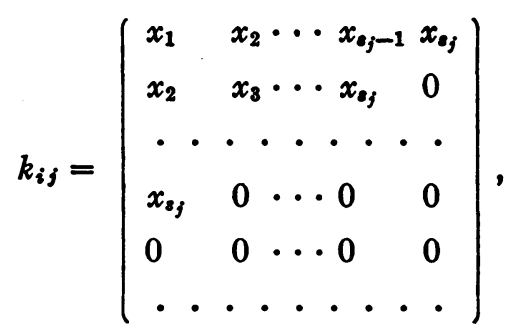

which contains $s_{i}$ rows, if $s_{j}<s_{i}$ with addition of a sufficient number of zero rows, and which contains $2 s_{i}$ parameters (a complex number is counted as two parameters). Further in case $i=j$ evidently $k_{i i}$ equals zero.

Therefore $K$ depends on

$$
\begin{gathered}
2 s_{2}+2 s_{3}+\cdots+2 s_{\bullet} \\
+2 s_{3}+\cdots+2 s \\
\cdots \\
+2 s \\
=2 s_{2}+4 s_{3}+\cdots+2(e-1) s_{0}
\end{gathered}
$$

parameters.

10. The case with a positive root. Now we take

$$
\begin{aligned}
& S=\sum_{1 \leqq i \leqq o}^{\prime}\left( \pm j^{\left(\theta_{i}\right)}\right), \\
& H=\sum_{1 \leq i \leq e}^{\prime}\left( \pm j j_{\alpha}^{\left(\delta_{i}\right)}\right),
\end{aligned}
$$

where $s_{1} \geqq s_{2} \geqq \cdots \geqq s_{\text {e. }}$ Let

$$
P=(I+Q)(I-Q)^{-1} .
$$

We find, from (34) and (35), that

$$
Q S+S Q^{\prime}=0,
$$

and

$$
\bar{Q} H+H Q^{\prime}=0 .
$$

Putting $Q S=K$, which is skew symmetric, we have 


$$
\bar{K} S^{-1} H-H S^{-1} K=0 .
$$

Since $\alpha \neq-\alpha$, we find that $K$ is real. Let

$$
K=\left(k_{i j}\right), \quad k_{i j}=k_{i j}^{\left(e_{i}, \theta_{j}\right)} \text {. }
$$

Then

$$
\bar{k}_{i j} j_{\alpha}^{\left(e_{j}\right)}=\left(j^{\left(e_{j}\right)} j_{\alpha}^{\left(e_{i}\right)} j^{\left(e_{i j}\right)}\right) k_{i j}=j^{\left(e_{i}\right)^{\prime}} k_{i j}
$$

In a manner similar to that of $\S 9$, it can be shown that the number of parameters is equal to

$$
2 s_{2}+4 s_{8}+\cdots+2(e-1) s_{\text {. }}
$$

11. The case with a negative root. Now we may let

$$
\begin{aligned}
& S=\sum_{1 \leqq i \leqq \bullet}\left(\begin{array}{cc}
j^{\left(s_{i}\right)} & 0 \\
0 & j^{\left(\left(_{i}\right)\right.}
\end{array}\right), \\
& H=\sum_{1 \leqq i \leqq \bullet} i\left(\begin{array}{cc}
0 & j j_{\beta}^{\left(\theta_{i}\right)} \\
-j j_{\beta}^{\left(s_{i}\right)} & 0
\end{array}\right)
\end{aligned}
$$

where $\beta>0$. Let

$$
J=\sum_{1 \leqq i \leqq}^{\prime} j^{\left(\theta_{i}\right)}, \quad J_{\beta}=\sum_{1 \leqq i \leqq o}^{\prime} j^{\left(s_{i}\right)} j_{\beta}^{\left(\theta_{i}\right)} .
$$

We may consider, without loss of generality, the case

$$
S=\left(\begin{array}{ll}
J & 0 \\
0 & J
\end{array}\right), \quad H=i\left(\begin{array}{cc}
0 & J_{\beta} \\
-J_{\beta} & 0
\end{array}\right) .
$$

Letting

$$
P=(I+Q)(I-Q)^{-1}
$$

and

$$
Q\left(\begin{array}{ll}
J & 0 \\
0 & J
\end{array}\right)=K,
$$

which is skew symmetric, we find, from (36), that

$$
\bar{K}\left(\begin{array}{cc}
0 & J J_{\beta} \\
-J J_{\beta} & 0
\end{array}\right)=\left(\begin{array}{cc}
0 & J_{\beta} J \\
-J_{\beta} J & 0
\end{array}\right) K .
$$

Let

$$
K=\left(\begin{array}{cc}
K_{1} & L \\
-L^{\prime} & K_{2}
\end{array}\right)
$$

From (44), we have 


$$
\begin{aligned}
\bar{L} J J_{\beta} & =J_{\beta} J L^{\prime}, & \bar{L}^{\prime} J J_{\beta} & =J_{\beta} J L, \\
\bar{K}_{1} J J_{\beta} & =J_{\beta} J K_{2}, & \bar{K}_{2} J J_{\beta} & =J_{\beta} J K_{1} .
\end{aligned}
$$

Putting $\bar{L} J J_{\beta}=T$, which is Hermitian by (46), we have

$$
\begin{aligned}
T\left(J J_{\beta}\right)^{2} & =\left(J_{\beta} J\right)^{2} T, \\
K_{1}\left(J J_{\beta}\right)^{2} & =\left(J_{\beta} J\right)^{2} K_{1}
\end{aligned}
$$

and

$$
K_{2}=\left(J_{\beta} J\right)^{-1} \bar{K}_{1}\left(J J_{\beta}\right) .
$$

Consequently, for $T$ and $K_{1}$ satisfying (48) and (49) and $K_{2}$ defined by (50), we have $K$ defined by (45) to meet our requirement.

The number of parameters of $K_{1}$ is equal to

$$
2 s_{2}+4 s_{3}+\cdots+2(e-1) s_{e},
$$

and the number of $T$ is equal to

$$
2 s_{2}+4 s_{3}+\cdots+2(e-1) s_{\bullet}+s_{1}+s_{2}+\cdots+s_{\text {e. }}
$$

The total number of parameters of $K$ is, therefore, equal to

$$
s_{1}+5 s_{2}+9 s_{3}+\cdots+(4 e-3) s_{\text {e. }}
$$

12. Automorphs (continuation). As a consequence of the previous results we have the following statement: Let the roots of $H S^{-1} \bar{H} \bar{S}^{-1}$ be

$$
\alpha_{1}, \cdots, \alpha_{\lambda}
$$

with the multiplicities

$$
\begin{gathered}
p_{1}, \cdots, p_{\lambda} ; \\
\beta_{1}, \cdots, \beta_{\mu} ; \quad \bar{\beta}_{1}, \cdots, \bar{\beta}_{\mu}
\end{gathered}
$$

with the multiplicities

$$
q_{1}, \cdots, q_{\mu} ; \quad q_{1}, \cdots, q_{\mu}
$$

and

$$
\gamma_{1}, \cdots, \gamma_{v}
$$

with the multiplicities

$$
2 r_{1}, \cdots, 2 r_{\text {, }}
$$

respectively. Then the group of automorphs of $(H, S)$ depends on at least

$$
r_{1}+r_{2}+\cdots+r_{2}
$$

parameters. (Evidently, this is a best possible constant.) 
TheOREM 6. Given $\lambda$ and an Hermitian matrix $H$, the symmetric matrices $S$ satisfying

$$
d\left(H S^{-1} \bar{H} \bar{S}^{-1}-\lambda I\right)=0
$$

depends on $n(n+1)-1, n(n+1)-2$, and $n(n+1)-3$ parameters according as $\lambda$ is positive, complex, and negative respectively.

Proof. The group of conjunctive automorphs of $H$, that is, the group formed by $\Gamma$ satisfying

$$
\bar{\Gamma} H \Gamma^{\prime}=H,
$$

depends on $n^{2}$ parameters. Now we ask what is the number of parameters of distinct

$$
\Gamma S \Gamma^{\prime} .
$$

Since the matrix $\Gamma$ satisfying

$$
\bar{\Gamma} H \Gamma^{\prime}=H, \quad \Gamma S \Gamma^{\prime}=S
$$

depends on not more than $r_{1}+\cdots+r_{\nu}$ parameters, if the roots of $H S^{-1} \bar{H} \bar{S}^{-1}$ are given at the beginning of the section, the totality of different symmetric matrices $\Gamma S \Gamma^{\prime}$ depends on $n^{2}-r_{1}-\cdots-r_{\nu}$ parameters.

For a given positive $\lambda$, the manifold formed by $S$ (varying all the other roots) depends on $n^{2}+(n-1)$ parameters. Similarly we have the result for a complex root.

For a given negative root, the number of parameters of other roots is equal to $n-2$. Further the different symmetric $\Gamma S \Gamma^{\prime}$, for all roots being given, depends on at most $n^{2}-1$ parameters. (In case all the others are non-negative $n^{2}-1$ is the exact number.) Thus the total number of parameters is equal to $n^{2}-n-3$.

Finally, the author wishes to express his warmest thanks to the referee for his help with the manuscript.

National Tsing Hua University, Kunming, Yunnan, China. 